\section{artelogie}

\section{Artelogie}

Recherche sur les arts, le patrimoine et la littérature de I'Amérique latine

10 | 2017

Après le paysage : l'art, l'inscription et la représentation de la nature en Amérique latine aujourd'hui

\title{
Voyage pittoresque et historique au Brésil, de Jean- Baptiste Debret, Jacques Leenhardt (préface)
}

\section{Raphael Fonseca}

\section{OpenEdition}

\section{Journals}

Édition électronique

URL : http://journals.openedition.org/artelogie/936

DOI : 10.4000/artelogie.936

ISSN : 2115-6395

Éditeur

Association ESCAL

Référence électronique

Raphael Fonseca, « Voyage pittoresque et historique au Brésil, de Jean-Baptiste Debret, Jacques Leenhardt (préface) », Artelogie [En ligne], 10 | 2017, mis en ligne le 05 avril 2017, consulté le 23 septembre 2020. URL : http://journals.openedition.org/artelogie/936 ; DOI : https://doi.org/10.4000/ artelogie.936

Ce document a été généré automatiquement le 23 septembre 2020

Association ESCAL 


\title{
Voyage pittoresque et historique au Brésil, de Jean-Baptiste Debret, Jacques Leenhardt (préface)
}

\author{
Raphael Fonseca
}

\section{RÉFÉRENCE}

Jean-Baptiste Debret, Jacques Leenhardt (prefacio), Viagem pitoresca e histórica ao Brasil, São Paulo, Imprensa Oficial do Estado de São Paulo, 2016, 652p.

1 Le temps des commémorations et du souvenir a marqué cette année 2016 dans le champ des études de l'art et de l'image au cours du XIX ${ }^{\mathrm{e}}$ siècle au Brésil. Les deux cents ans de la venue d'un groupe d'artistes français en 1816 à Rio de Janeiro - alors capitale de l'empire luso-brésilien - étaient au centre des célébrations. Baptisé a posteriori "Mission artistique française», ce voyage se définit comme la somme de deux souhaits : la sortie du territoire français d'artistes alors politiquement associés à un Napoléon sur sa fin politique imminente et le désir de l'empereur portugais Jean VI d'établir une École des Beaux-Arts sur le territoire brésilien. Il est important de rappeler que le Portugal ne possédait pas de telles institutions en Europe pourtant, la présence de ces artistes au Brésil permettait d'aider au développement d'une culture visuelle imprégnée de la tradition classique européenne (et de répertoires iconographiques impériaux) au cœur même des tropiques.

Dans ce désir de reconsidération de ce bicentenaire, des événements et des publications ont été organisés autour de ces artistes qui sont venus au Brésil et autour des conséquences de cette présence sur l'enseignement des arts visuels dans le pays. L'une des publications les plus récentes n'est autre que la réédition d'un ouvrage classique Voyage pittoresque et historique au Brésil, écrit par l'artiste français Jean-Baptiste Debret (1768-1848) et présenté en 2015 par l'éditeur Imprensa Oficial do Estado de São Paulo. Attirant l'attention des médias spécialisés, le livre a fait l'objet d'un événement 
organisé par la Casa de Rui Barbosa à Rio de Janeiro, intitulé «200 ans depuis la découverte du Brésil par Jean-Baptiste Debret». Dirigée par le professeur Jacques Leenhardt, directeur de l'École des Hautes Études en Sciences Sociales de Paris, l'organisation de ce livre est similaire à la troisième édition en portugais des textes écrits par Debret sur son séjour au Brésil.

Né à Paris en 1768 et mort dans cette même ville en 1848, Debret était étudiant à l'École des Beaux-Arts et possédait une forte formation classique.

4 Cousin de Jacques-Louis David, il était spécialiste de peinture d'histoire et reçu le Prix de Rome en 1791. Outre les commandes de portraits et de peintures d'histoire pour Jean VI et son fils, Pierre $I^{\mathrm{er}}$, Debret se consacre, dès son arrivée au Brésil en 1816, à la peinture de scènes urbaines de Rio de Janeiro. L'artiste réalise une grande série d'images abordant le quotidien de la capitale. Les relations de pouvoir, le travail, la présence essentielle des esclaves africains et des communautés indigènes sont quelques-uns des grands groupes d'illustrations qu'il réalise. Ces petits formats qui ne dépassaient guère les trente centimètres de large sont, pour Debret, l'occasion d'appliquer les techniques du dessin et de l'aquarelle afin de réussir à transposer sous forme d'historiettes la vie quotidienne dans cette partie des tropiques.

5 Parallèlement, Debret, accompagné d'autres artistes français qui ont entrepris ce voyage au Brésil, comme Grandjean de Montigny, Nicolas-Antoine Taunay et Joachin Lebreton, définissent progressivement les contours d'un projet de création d'une École Royale des Sciences, Arts et Métiers, future Académie Impériale des Beaux-Arts. Si l'édifice de l'Académie fut érigé en 1822, son inauguration n'eut lieu qu'en 1826, soit dix ans après l'arrivée du groupe. A cette date, Debret occupait la chaire de peinture d'histoire et le poste de directeur de l'institution de 1828 à 1831, année de son retour en Europe.

6 En 1834, il réunit en France cent-cinquante-trois de ses aquarelles et les transforme en lithographies, en assurant lui-même l'opération technique. De cette sélection, naît un album qui diffuse en Europe, à la fois, les images d'un Brésil en pleine expansion et en adéquation avec la présence de la cour portugaise, et celles liées à un imaginaire dévoilant un territoire sauvage et vierge. Debret se chargea aussi de l'analyse textuelle de la société brésilienne. Il ne s'agissait pas d'un quelconque voyage, mais d'un déplacement qui, relaté à travers ses propres mots, prenait un ton pittoresque et historique : le contraste entre l'Europe et l'Amérique était maintenu, soulignant les particularismes locaux dignes « d'être peints », non sans un compromis historique où la jeune Histoire du Brésil était commentée par des documents et par le regard critique de Debret.

Divisé en trois volumes, le livre repose sur une linéarité discursive très claire. Le premier volume a trait aux groupes indigènes que l'auteur observa très certainement lors de son séjour brésilien et qu'il « documente » à travers des illustrations. Différents groupes d'indigènes sont transposés en image et en mots, avec certains chapitres exclusivement dédiés aux divers objets (comme les paniers, les céramiques et les armes), en sus d'une carte du Brésil placée à la fin du volume. Le second volume regroupe les us et coutumes des espaces plus urbains du Brésil qui se rapprochent de l'expérience vécue par Debret, en grande partie, à Rio de Janeiro. Enfin, un contraste s'opère alors entre les deux premiers volumes: d'un côté, l'indigène vu dans ce qui restait de son milieu «naturel » à travers une clef de lecture primitiviste mais tout aussi attentive aux indigènes « acculturés »; de l'autre, les descriptions du quotidien de 
la capitale de l'empire, où il y avait une profonde division entre esclaves noirs, immigrants européens et propriétaires d'établissements commerciaux. Outre les descriptions vestimentaires et les cortèges militaires, le troisième et dernier volume est consacré aux rituels religieux (mariages, enterrements et fêtes publiques).

8 Si l'arrivée de Debret en 1816 coïncide avec la mort de la reine du Portugal, Marie I ${ }^{\mathrm{er}}$, il travaille déjà au couronnement de Jean VI. À son départ du Brésil en 1831, il quitte un empire indépendant du gouvernement portugais, avec, à sa tête, Pierre $\mathrm{I}^{\mathrm{er}}$, alors prêt à retourner au Portugal et à abdiquer en faveur de son fils, Pierre II. Durant ces quinze années, la politique brésilienne naît donc sous les yeux de Debret, avides d'observer le développement d'une civilisation. Dans son ouvrage, Debret tente alors de rendre compte d'un processus d' " occidentalisation » du Brésil. Comme toute tentative, il était impossible de ne pas souligner, ni même de démontrer par l'image, les aspects ne cadrant pas avec le modèle d'une société civilisée, telle qu'il la connaissait en France. Comment ne pas exagérer les aspects culturels d'un territoire encore peuplé d'indigènes et où l'esclavage était la pierre angulaire du développement du pays ?

9 La nouvelle publication en portugais du livre de Debret est importante dans son immédiateté au regard de l'écart chronologique avec les autres éditions : la première fut réalisée par Sérgio Milliet dans les années 1940 et la seconde a été éditée à la fin des années 1980. En d'autres termes, cela faisait vingt ans qu'une édition était attendue afin de rendre les écrits de Debret accessibles sur le marché éditorial brésilien. Tout chercheur spécialisé connaît en effet les difficultés rencontrées pour trouver ou acheter les éditions antérieures de ce texte et ce nouvel ouvrage remplit pleinement son rôle.

10 La première traduction faite de ce texte en portugais par Milliet a justement jeté les bases de ce livre. Une mise à jour de la langue portugaise a été réalisée en raison des soixante-dix années écoulées entre les deux publications. Le directeur Jacques Leenhardt - responsable entre autres de la première édition française de l'ouvrage - a choisi non seulement de participer à l'édition du texte mais aussi de rédiger un article important relatif à la production et au parcours de Debret. Intitulé « Un regard décalé sur la construction de la nation brésilienne ", l'auteur analyse les altérations formelles et les angles d'approches employés pour appréhender le quotidien qui, dans les œuvres de Debret, ont souffert de ce déplacement de la France au Brésil.

11 Ainsi, dans toutes publications quelles qu'elles soient, $\mathrm{y}$-a-t-il toujours des éléments semant le doute dans l'esprit du chercheur? En premier lieu, il est important de mettre l'accent sur le choix éditorial de rassembler en un même ouvrage les trois tomes de Debret. Au lieu de consulter séparément les trois volumes (qui ont été respectivement publiés en 1834, 1835 et 1839), le lecteur a entre les mains les trois parties regroupées en un tout. Ce choix aurait été judicieux si l'ouvrage de 652 pages en format A4 ne pesait pas quatre kilos au point que le porter et le feuilleter en devient presque fatiguant.

12 Ces choix de format d'impression engendrent un autre facteur délicat dans le cadre du marché éditorial brésilien et de la réalité socio-économique du pays : le livre coûte très cher et dépasse le seuil des deux cents reais, ainsi qu'il est indiqué sur le site de la maison d'édition. Ceci pourrait se justifier par la nature de l'audience ciblée, à savoir les lecteurs spécialisés dans le domaine des arts visuels (et qui auraient peut-être les moyens suffisants pour l'acheter). Toutefois, cette ligne de pensée ne contribue en aucun cas à la diffusion, ni même à la vulgarisation de l'œuvre de Debret. La valeur que 
possède cette publication au regard de l'accessibilité du texte actualisé fait face un obstacle, celui du coût. Celui-ci ne sera certainement pas proportionnel au budget d'une grande partie des jeunes chercheurs, universitaires et lecteurs néophytes susceptibles de s'intéresser aux écrits du Français.

Enfin, pour ce qui est de la pagination et du design, le directeur de l'ouvrage et l'éditeur ont choisi d'utiliser les gravures aquarellées de Debret afin de rendre effective la relation image-texte à travers ses mots. Cette option est intéressante et diffère des éditions antérieures du livre mais elle soulève la question de savoir s'il n'était pas plus pertinent d'utiliser une édition en noir et blanc, alors plus fidèle à ce qui devait largement circuler en Europe à ce moment-là. Le grand format des images participe à une lecture du texte sans interruption, en d'autres termes, parfois, certains segments du texte séparés dans la version originale et qui permettaient d'établir une relation avec les images, sont, dans la présente édition, regroupés sur une même page. Les images ne sont dès lors plus associées directement au texte mais poussent le lecteur à feuilleter l'ouvrage à la recherche du texte ou de l'image correspondant.

Ces observations sur le livre n'enlèvent en rien de son importance eu égard à l'état actuel des publications de sources primaires au Brésil, tant pour les études sociologiques qu'historico-artistiques. Bien qu'elle soit étudiée et citée par divers chercheurs spécialisés dans les relations entre art, image et identité au Brésil, l'œuvre de Jean-Baptiste Debret est exclusivement abordée sous l'angle de sa production imagière, laissant de côté la relation essentielle entre son regard d'artiste et l'Autre, ainsi que ses commentaires le concernant. Ces quelques petits ajustements formels apporteraient peut-être au public intéressé un résultat plus fidèle aux premières versions en langue française. Cet aspect ajouté à une réévaluation du format et du coût permettrait d'étendre sa portée et sa qualité en tant que livre.

Une version numérique du livre - encore inexistante - serait certainement une manière moins chère et plus abordable d'acheter une publication au tirage encore restreint à un nombre précis d'exemplaires. En outre, le format numérique invite à une recherche plus transversale, permettant ainsi aux mots de Debret de gagner en résonance non seulement à travers un intérêt porté sur son ensemble mais aussi pour les lecteurs désireux de rechercher un thème spécifique au cœur de ce livre monumental. Fort heureusement, les images et les phrases de Debret possèdent toujours autant de puissance et de poids et ce, deux-cents ans après sa venue au Brésil, invitant le lecteur à de nouvelles perspectives, à de nouveaux croisements et à des études novatrices.

\section{AUTEURS}

\section{RAPHAEL FONSECA}

Doutor em História da arte, Universidade Federal de Rio de Janeiro. raphaelfonseca[at]gmail.com 